\title{
Space electromagnetic interference analysis of secondary equipment signal cable
}

\author{
Fan Kuang ${ }^{1 *}$, Yunfeng Xia ${ }^{1}$, Tianyu Cheng ${ }^{1}$, Rongfu Zhong ${ }^{1}$, Haikun Wei ${ }^{1}$ \\ ${ }^{1}$ Dongguan Power Supply Bureau of Guangdong Power Grid Corporation, Dongguan 523000, China
}

\begin{abstract}
In the field of high-voltage transmission and distribution, the secondary equipments are influenced by the electromagnetic coupling effect generated by the primary circuit, which causes strong interference to the signal cable. In this paper, an equivalent circuit model is established for the secondary cables in different forms, namely conventional cable, coaxial cable and twisted pair cable, based on transmission line theory. Based on the model, the responses of these three kinds of signal cables under the impact of spatial plane electromagnetic wave are analysed in frequency domain. Different impedance characteristics of the cables at the near-end and far-end, and different cable layouts, and different injection directions of the electromagnetic wave are considered in the analysis. The response characteristics of different kinds of signal cables subjected to electromagnetic wave are obtained in frequency domain. By comparing the response characteristics of different kinds of signal cables in different conditions, measures to supress the interference from the coupling of electromagnetic wave are obtained. It can be used to guide the selection and cabling of the signal cables for the secondary equipment in power system.
\end{abstract}

\section{Introduction}

In recent years, with the large-scale construction of smart grid, more and more secondary equipments such as electronic devices, smart meters and smart devices are applied in the field of power transmission and distribution. Due to the complex and harsh electromagnetic environment in high-voltage transmission and distribution or substation, the signal cables on the secondary circuit will suffer severe interference problems through electromagnetic coupling. With the increasing of the capacity and voltage level of the power system, the electromagnetic interference generated by primary circuit is more and more strong. What's more, as the secondary equipments are becoming more miniaturized, digitized and electronic, they are more susceptible to the influence of electromagnetic interference. Especially in the high voltage switch environment, the signal cables between control room and secondary equipments are more vulnerable to serious electromagnetic interference.

Electromagnetic wave can transmit energy to the connected equipment through the coupling of signal cable. If the power of the radiated electromagnetic wave is very large, it will generate a significant impact on the secondary system. Therefore, it is of great value to analyse the characteristics of the interference from the radiated electromagnetic wave and provide theoretical guarantee for the normal operation of electronic and electrical equipment.

Generally, there are two main methods to analyse the problem of interference caused by spatial electromagnetic interference, namely field method and the equivalent circuit method. The field method is based on Maxwell equation. It is used to solve the boundry value of the inner and outer field of the cable system under given boundary conditions. The equivalent circuit method is on the basis of the transimission line theory. It is used to solve the response of external electromagnetic field inside the cable by equivalent the field-circuit coupling problem of spatial electromagnetic field to signal cable to the transmission line model[1].

Theoretically, the field method is very strict and has accurate calculation results. However, due to the special complexity of the calculation process and high requirements on the computer, there are some difficulties in practical application. In contrast, the equivalent circuit method is simple and practical in engineering applications because of its fast computing speed. Therefore, the equivalent circuit method is used to analyze the spatial electromagnetic interference of signal cables of secondary equipments in this paper.

When solving the field-circuit coupling problem, the transmission line theory mainly includes three models, the Taylor model[2] containing distributed voltage source and distributed current source, the Agrawal model[3] containing only distributed voltage source and the Rachidi model[4] containing only distributed current source. Different transmission line models are solved mainly through time-domain analysis and frequency-domain analysis [5]. In this paper, the equivalent circuit method is adopted to quantitatively analyze and calculate the conventional signal cable, coaxial cable and twisted-pair cable of the secondary equipment. 


\section{Analysis of space electromagnetic field radiation interference}

\subsection{Analysis model}

In the analysis, it is assumed that the spatial electromagnetic radiation is in the form of a plane wave. Due to the linearity, the electric field intensity is assumed $E=1 \mathrm{~V} / \mathrm{m}$. As the free space characteristic impedance is $Z_{0}=377 \Omega$, the corresponding magnetic field intensity is $H=E / Z_{0}=2.65 \mathrm{~mA} / \mathrm{m}$ (corresponding to the magnetic flux density $B=3.3 \mathrm{nT}$ ). Considering the linear medium and calculating the interference in a unit length (i.e., a length of $1 \mathrm{~m})$, the real radiated interference can be estimated based on the calculation results of the above reference conditions and the actual stray field intensity.

The analysis model of the interference from radiated electromagnetic wave is shown in Fig. $1, N_{l}$ and $N_{2}$ are two nodes at both ends of the cable, where $N_{l}$ connects to the far-end of the circuit (e.g., secondary equipment control system), and $N_{2}$ connects to the the near-end of the circuit (such as the secondary equipment sensor or transducer). The length of the cable is assumed $1 \mathrm{~m}$. Then we take the direction of electric field $E$ parallel to the direction of the cable, and the direction of magnetic field $H$ perpendicular to the cable but parallel to the ground plane, and the direction of plane wave $S$ propagation perpendicular to the ground plane.

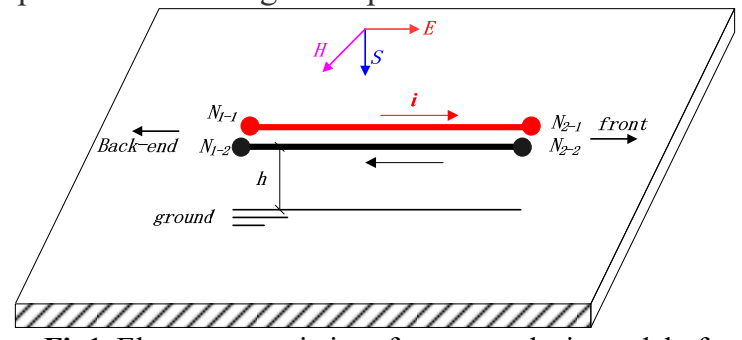

Fig1. Electromagnetic interference analysis model of conventional signal lines

\subsection{Interference of conventional signal cable}

In the analysis, the diameter and insulation thickness of the signal cable are $1.5 \mathrm{~mm}$ and $1 \mathrm{~mm}$, and three layouts of the cables as shown in Fig. 2 are taken into account. The distance of the cables from the ground is $h=1 \mathrm{~cm}$.

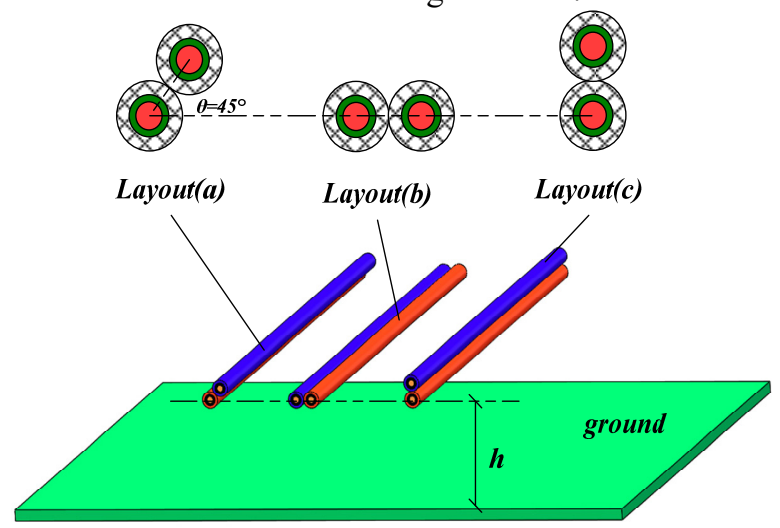

Fig2. Three layouts of conventional signal lines

Fig. 3 shows the equivalent circuit model of conventional signal cable. The cable contains two roots, the forward and the backward. Assuming that the cable is connected to the ground on both ends through $1 \mathrm{pF}$ stray capacitance, and both ends of the cable are connected with standard resistance $R=50 \Omega$. The differential-mode interference voltage signals received at both ends of the cable $\left(P_{1}\right.$ and $\left.P_{2}\right)$ are calculated, respectively.

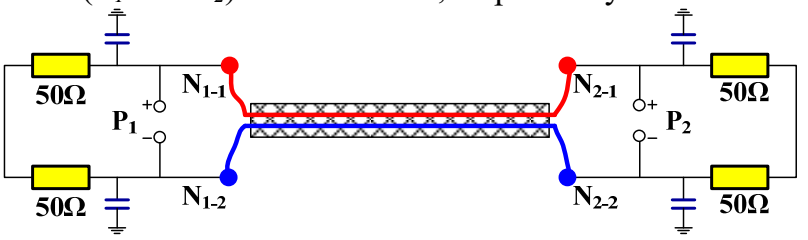

Fig3. Equivalent circuit model of conventional signal cable (standard impedance at both near-end and far-end)

The results of differential-mode voltage coupled by the conventional signal cable with standard impedance at both ends are shown in Fig. 4. It can be seen from the calculation results that the coupled differential-mode interference voltage basically increases linearly with the frequency within $100 \mathrm{MHz}$ in logarithmic coordinate. There are two resonance points around $120 \mathrm{MHz}$ and $370 \mathrm{MHz}$. The differential-mode interference of the three kinds of cable layouts shown in Fig. 2 are basically the same. The cable layout (b) has the lowest differentialmode interference, about 3 orders of magnitude less than other two cases, and the result of cable layout (c) is slightly higher than (a). It is because the electric fields in all three layouts are parallel to the cable, in other words, the interfering voltage introduced by the electric are the same. In contrast, the effect of the magnetic field on various configurations is different. In layout (c), it is the strongest, followed by layout (a), and layout (b) is nearly zero. That is because that the loop area affected by the magnetic field is the largest in layout (c). As the magnetic field is parallel to the cable plane in layout (b), the magnetic field has little impact on it..

At the highest resonance point, the differential-mode voltage under the condition of unit electric field and unit length reaches to $20 \mathrm{mV}$. But these resonance frequencies are beyond the frequency of concern. The interference voltage generated by $1 \mathrm{kHz}$ plane wave per unit strength on the cable with a unit length is about $1.6 \times 10^{-13} \mathrm{~V}$. Assuming the interference magnetic field is $1 \mathrm{mT}$, the differential-mode interference voltage corresponding to a unit length is about $48 \mathrm{nV}$. Then, assuming the transmission distance is $100 \mathrm{~m}$, the corresponding differential-mode interference voltage is about $4.8 \mu \mathrm{V}$, much lower than the general standard signal.

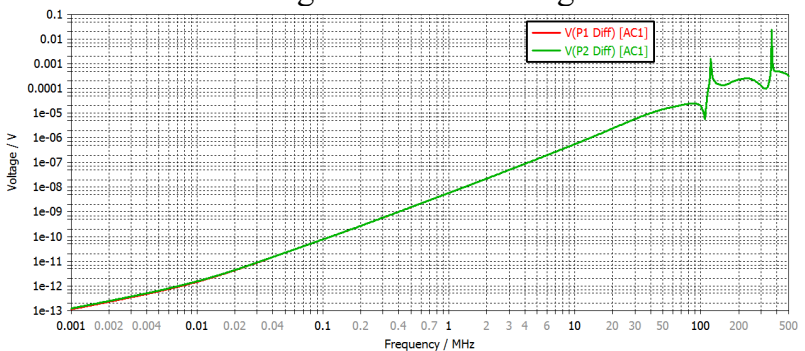

(a) 


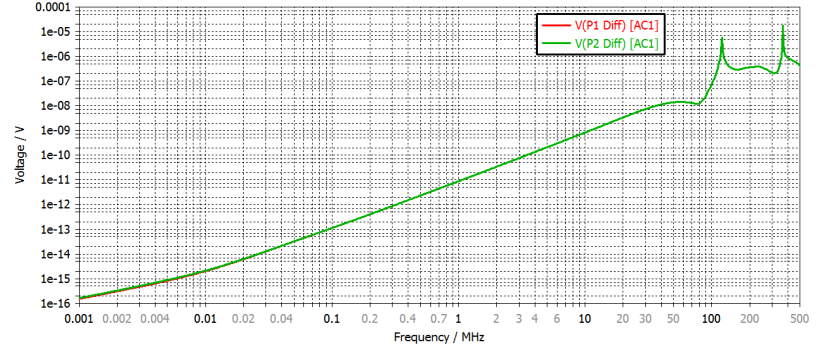

(b)

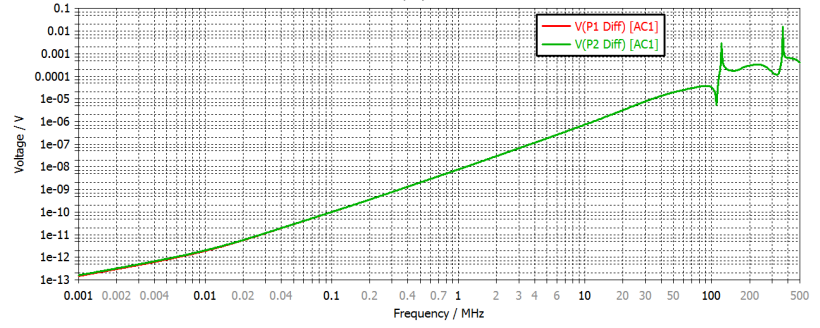

(c)

Fig4. Radiation-coupled differential-mode voltage of standard impedance of conventional signal cable (standard impedance at both near-end and far-end)

In the case of open at far-end, two resistances $R=1 \mathrm{M} \Omega$ are connected to the front-end to simulate this situation as shown in Fig. 5. The results of differential-mode voltage between two ports of conventional signal cables are shown in Fig. 6. The differential-mode interference voltage spectrum characteristics are similar to the ones in Fig. 4. However, due to the open-circuit state at the far-end, there is a certain difference between the voltage at near-end and the far-end. The voltage at the far-end is increased to some extent. The interference voltage generated by $1 \mathrm{kHz}$ plane wave per unit strength on a unit length cable rises to $3.3 \times 10^{-13} \mathrm{~V}$. At the same time, the peak differential-mode voltage at the resonance point reaches $40 \mathrm{mV}$, which is double of the previous ones.

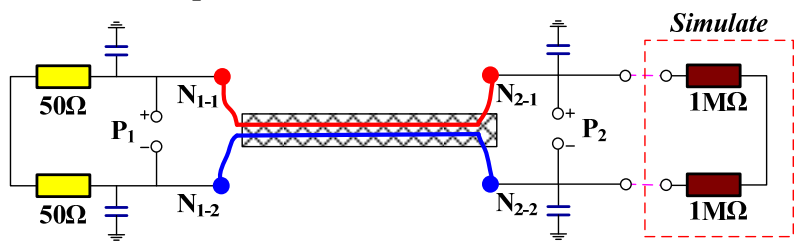

Fig5. Equivalent circuit Model of conventional signal cable (standard impedance at near-end and open at far-end)

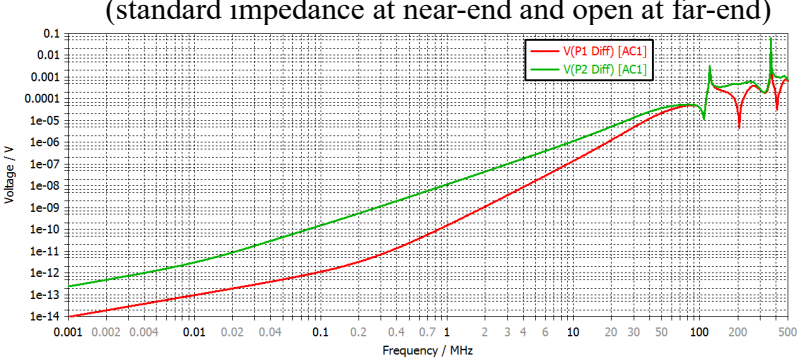

(a)

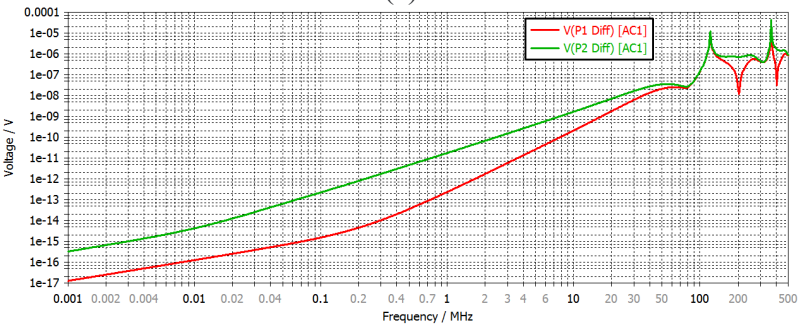

(b)

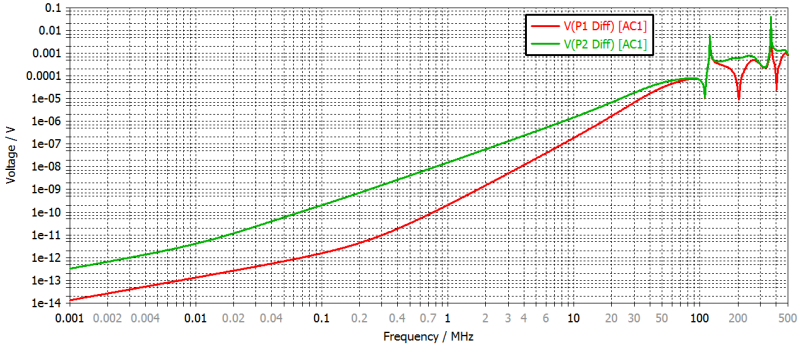

(c)

Fig6. Standard impedance radiation-coupled differential-mode voltage for conventional signal cables (standard impedance at near-end andopen at far-end

\subsection{Interference of coaxial cable}

For coaxial cables, the physical model of cable layout is similar to the one in Fig.2. But the equivalent circuit model is slightly different, as shown in Fig.7. The far-end is connected to standard resistors, and the near-end of the circuit is grounded.

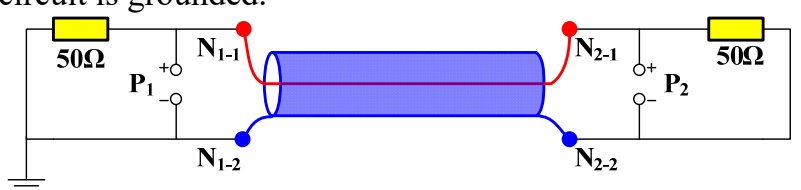

Fig7. Equivalent circuit model of coaxial cable (standard impedance at both near-end and far-end)

The differential-mode voltage analysis results of coaxial cable are shown in Fig. 8. According to the results, the differential-mode interference voltage of coaxial cable at low frequency is higher than that of conventional cable. The interference voltage generated by $1 \mathrm{kHz}$ plane wave per unit strength on unit length cable is about $1.5 \times 10^{-12} \mathrm{~V}$, which is about 10 times that of conventional cable. However, at high frequencies, the differential-mode interference voltage of coaxial cable is lower, and there are more resonance frequency points. The peak differentialmode interference voltage among the resonance points is about $4 \mathrm{mV}$, which is about $1 / 5$ of that of conventional cable (Fig. 4). At $1 \mathrm{MHz}$, the differential-mode interference voltage of conventional cables is about $5.7 \mathrm{nV}$, while that of coaxial cables is about $2.1 \mathrm{nV}$.

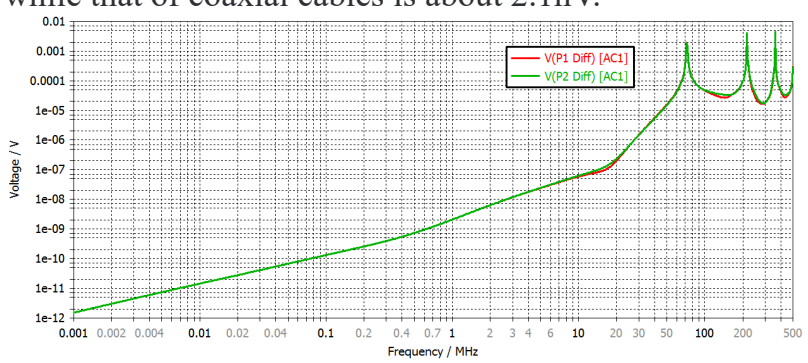

Fig8. Radiation-coupled differential-mode voltage of coaxial cable (standard impedance at both near-end and far-end)

The equivalent circuit of coaxial cable open at far-end is shown in Fig. 9. The result of differential-mode interfenrence voltage at two ports is shown in Fig. 10. According to the result, differential-mode interference voltage spectrum characteristics are similar to Fig. 8. As the circuit is open at the far-end, the voltage at the nearend is different from that at the far-end, and the voltage at the far-end is increased. At $1 \mathrm{kHz}$, the corresponding frontend interference differential-mode voltage rises to $3 \times 10^{-}$ 
${ }^{12} \mathrm{~V}$. At the same time, resonance peak differential-mode voltage reachs to $8 \mathrm{mV}$, enhanced to about double of that in the coaxial cable with standard resistance.

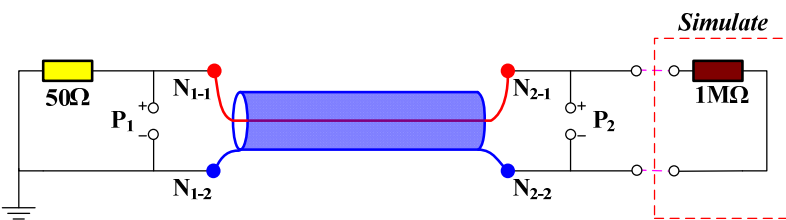

Fig9. Equivalent circuit model of coaxial cable (standard impedance at near-end and open at far-end)

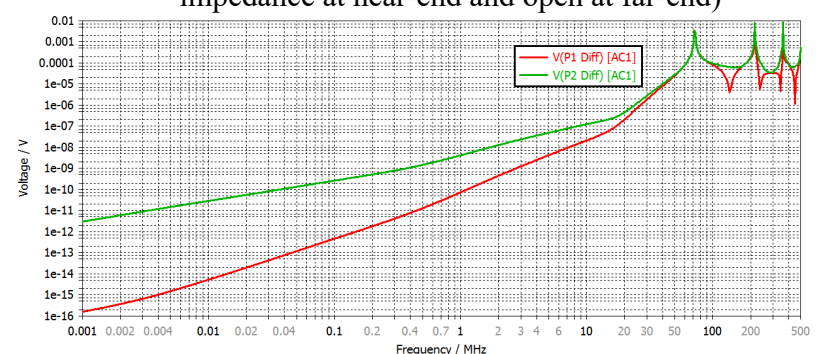

Fig10. Radiation-coupled differential-mode voltage of coaxial cable (standard impedance at near-end and open at farend)

\subsection{Interference of twisted-pair cable}

For twisted-pair cables, the equivalent circuit is shown in Fig. 11. Both ends are connected to standard impedance, and connected to the ground through stray capacitor.

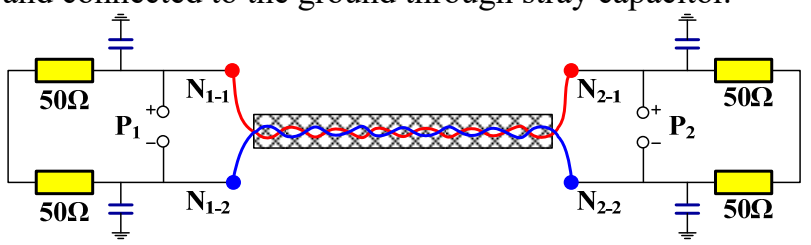

Fig11. Equivalent circuit Model of twisted pair (standard impedance at both ends)

The results of differential-mode voltage at two ports of twisted pair are shown in Fig. 12. According to the calculated results, the differential-mode interference voltage of twisted pair at low frequency is higher than that of conventional cable and coaxial cable. At $1 \mathrm{kHz}$, the differential-mode interference voltage per unit length is about $2.3 \times 10^{-11} \mathrm{~V}$, which is about 140 times that of conventional cable and 15 times that of coaxial cable. However, in high frequency range, the differential-mode interference voltage of twisted-pair cable is lower, and there are more resonance frequency points than other cables. The peak differential-mode interference voltage of the resonance points is about $0.5 \mathrm{mV}$, which is about $1 / 40$ of that of conventional cable and $1 / 8$ of that of coaxial cable. At $1 \mathrm{MHz}$, the differential-mode interference voltage of the twisted pair is $23 \mathrm{nV}$, that of the conventional cable is about $5.7 \mathrm{nV}$, and that of the coaxial cable is about $2.1 \mathrm{nV}$. The twisted pair has the highest differential-mode voltage, which indicates that the twisted-pair cablehas certain advantages only when it is above $1 \mathrm{MHz}$.

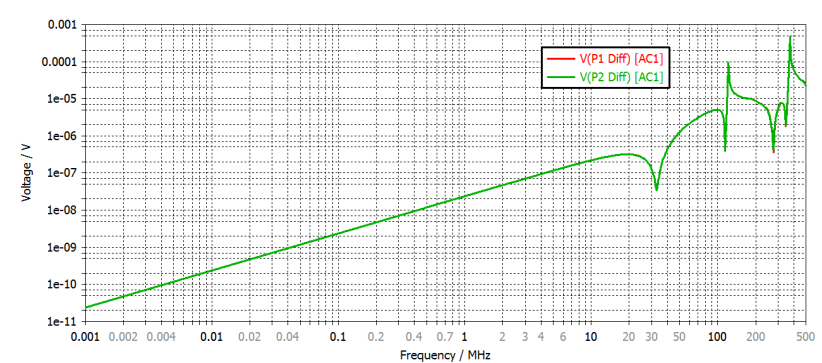

Fig12. Radiation-coupled differential-mode voltage of twisted pair (standard impedance at both ends)

The equivalent circuit of the twisted-pair cable open at far end, which is equivalent to $1 \mathrm{M} \Omega$ resistor, is shown in Fig. 13. The results of differential-mode voltage between two ports of the signal cables are shown in Fig. 14. According to the result, differential-mode interference voltage spectrum characteristics are similar to Fig. 12. At $1 \mathrm{kHz}$, the corresponding far-end differential-mode voltage rises to $4.7 \times 10^{-11} \mathrm{~V}$, and the resonance peak differentialmode voltage reaches to $1 \mathrm{mV}$, which is about doubled.

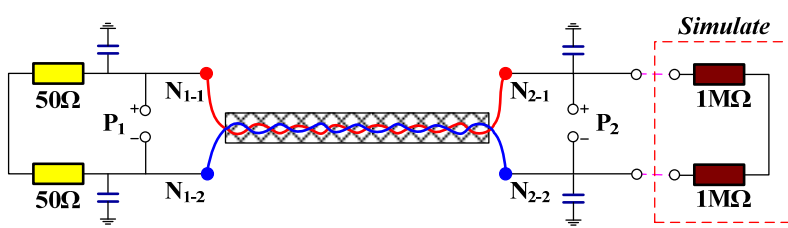

Fig13. Equivalent circuit Model of twisted pair(standard impedance at near-end and open at far-end)

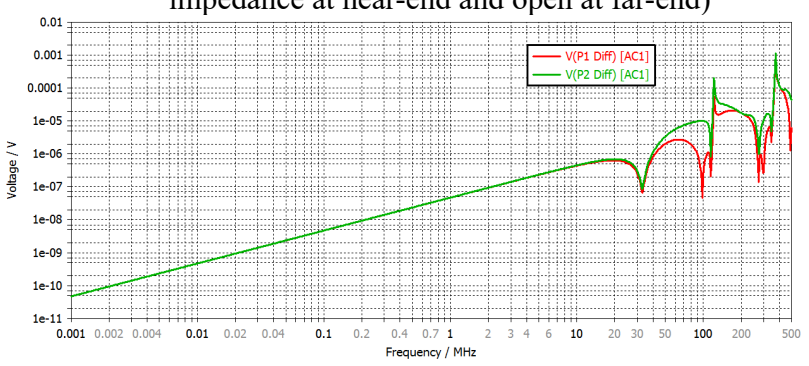

Fig14. Radiation coupled differential-mode voltage of twisted pair (standard impedance at near-end and open at far-end)

\section{Analysis of influence of incident direction of electromagnetic wave}

The direction of the plane wave has a great influence on the interference voltage. Taking the conventional cable layout as shown in Fig. 2 as an example, the influence of three different electromagnetic wave incident directions on the interference voltage is analyzed as shown in Fig.15.
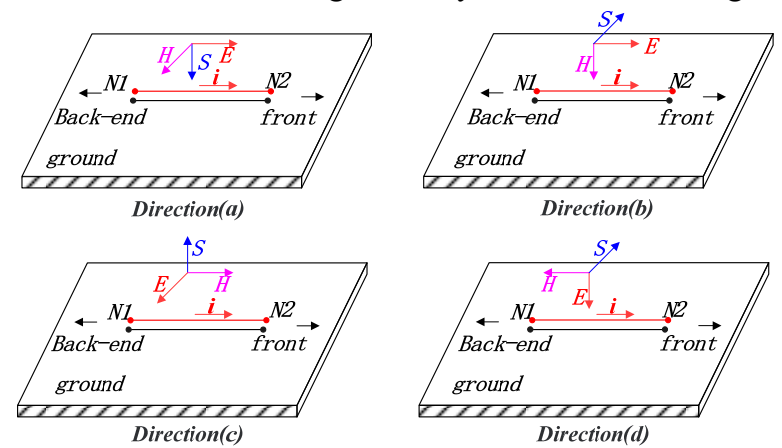

Fig15. Different incidence directions of electromagnetic waves

As shown in Fig. 16, the differential-mode voltage characteristics of (a) and (b) are very similar, but in high 
frequency band, the differential-mode voltage of (b) is lower, because the electric field effect of both is exactly the same. However, in (b), the direction of magnetic field $H$ is perpendicular to the ground plane, which will present a certain shielding performance. While in (a), there is no such effect. The differential-mode induced voltage of (c) is the smallest, which is six orders of magnitude lower than that of (a). This is because the electric field direction is perpendicular to the cable, and the magnetic field is parallel to the cable, so the differential-mode interference voltage introduced by both is small. The spectrum characteristics of the interferential voltage of (d) are similar to those of (b), which may be because the direction of the electric field $E$ is perpendicular to the ground plane, and the interference voltage is generated by the capacitance effect of the cable to the ground.

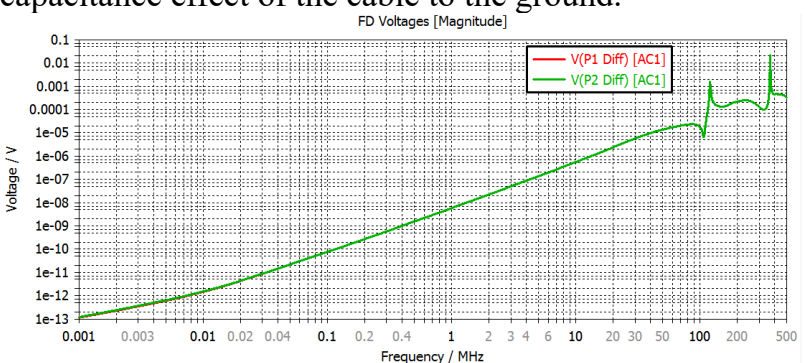

(a)

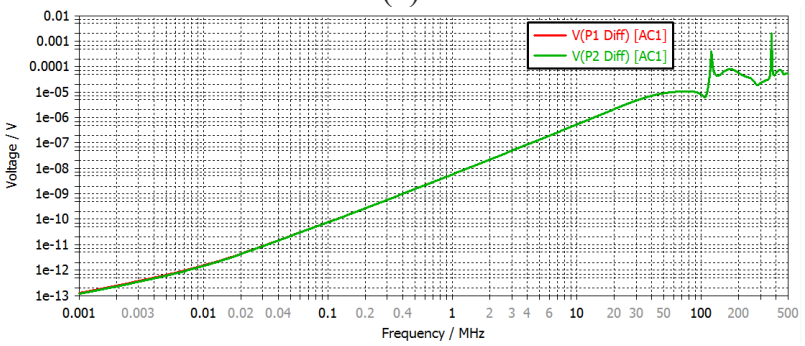

(b)

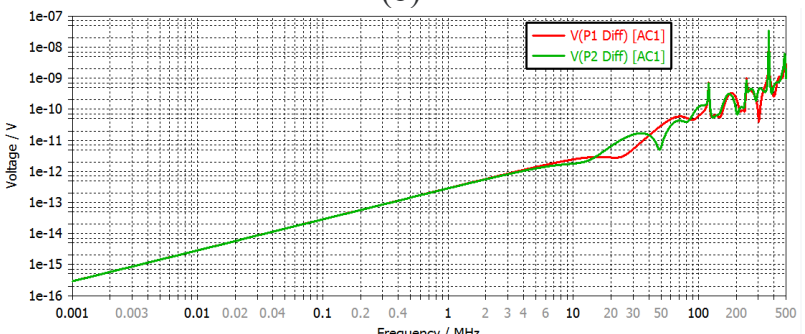

(c)

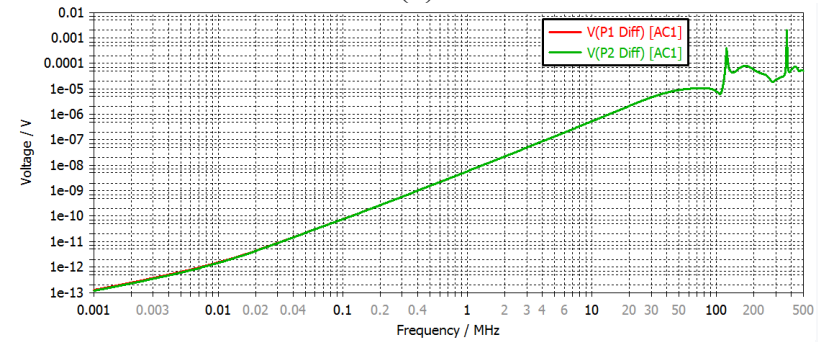

(d)

Fig16. Radiation-coupled differential-mode voltage of conventional cable under different plane wave incident directions(standard impedance at both ends)

\section{Conclusion}

This paper presents the analysis of the response characteristics of typical signal cables under the impact of electromagnetic wave. By comparing the response characteristics of different signal cables under different conditions, the following suggestions can be given to improve the immunity of secondary equipment in power system against the interference from electromagnetic wave.

(1) Coaxial cable and twisted-pair cable have a much better immunity than conventional cable. For sensitive or some important devices, coaxial cable or twisted-pair cable should be used.

(2) At high frequency (like higher than $1 \mathrm{MHz}$ ), twistedpair cable has a better behaviour than coaxial cable, while at low frequency (like lower than $1 \mathrm{MHz}$ ), the coaxial cable will behave better.

(3) If the direction of the electromagnetic wave can be predicted in advance, the cable is better arranged parallel with the magnetic field, but perpendicular to the electric field.

(4) At extremely severe conditions, shielding technique can be used to reduce the interference.

\section{References}

1. K. C. Chen, K. M. Daumrau. Accuracy of approximation transmission line formulas for overhead wires[J]. IEEE Trans. On EMC. 1989, 31(4):396 397.

2. Taylor C, Satterwhite R, Harrison Jr C. The response of a terminated two-wire transmission line excited by a nonuniform electromagnetic field[J]. IEEE Trans. On EMC. 1965,13(6):987 989.

3. Agrawal A K, Price H J, Gurbaxani S H. Transient response of multiconductor transmission lines excited by a nonuniform electromagnetic field[J]. IEEE Trans. On EMC. 1980(2):119 129.

4. Rachidi F. Formulation of the field-to-transmission line coupling equations in terms of magnetic excitation field[J]. IEEE Trans. On EMC. 1993,35(3):404 407.

5. Tesche F M, Karlsson T. EMC analysis methods and computation models[M]. JOHN Wiley and Sons, 1997. 